\title{
Esplenectomía para patologías hematológicas en la Fundación Santa Fe de Bogotá: caracterización y evaluación de múltiples desenlaces ${ }^{\S}$
}

\author{
Splenectomy for hematologic diseases at Fundación Santa Fe de Bogotá: \\ characterization and evaluation of multiple outcomes ${ }^{\S}$
}

\footnotetext{
'Grupo Hematología y Trasplante de Médula Ósea, Instituto de Oncología, Fundación Santa Fe de Bogotá (Bogotá, Colombia). Investigador asociado Hemo-ONCOLGroup.

${ }^{3}$ Facultad de Medicina, Universidad de los Andes (Bogotá, Colombia).

"Departamento de Laboratorio y Anatomía Patológica, Fundación Santa Fe de Bogotá (Bogotá, Colombia).

${ }^{5}$ Grupo Oncología Clínica y Traslacional, Instituto de Oncología, Fundación Santa Fe de Bogotá (Bogotá, Colombia).

${ }^{6}$ Departamento de Cirugía, Fundación Santa Fe de Bogotá (Bogotá, Colombia).

'Fundación para la Investigación Clínica y Molecular Aplicada del Cáncer (FICMAC); investigador asociado ONCOLGroup.
}

- Andrés Acevedo 1,2, Diana Cuervo33, Guillermo Quintero1,2, Myriam Rodríguez',2, Mónica Duarte', Martha Romero4, Carlos Saavedra ${ }^{4}$, Henry Becerra ${ }^{5}$, Carlos Castro ${ }^{5}$, Ricardo Nassar ${ }^{6}$, Andrés Felipe Cardona ${ }^{5,7}$

\begin{abstract}
Resumen
Introducción: La esplenectomía representa una herramienta diagnóstica y terapéutica para múltiples patologías hematológicas benignas y malignas. Se presenta el análisis de una cohorte de pacientes esplenectomizados por enfermedad hematológica en la Fundación Santa Fe de Bogotá.

Materiales y métodos: entre 2004 y 2011, se identificaron 30 pacientes esplenectomizados por indicación hematológica benigna $(n=23)$ y maligna $(n=7)$. Se evaluaron las historias clínicas respecto de diversos parámetros y desenlaces a largo plazo.

Resultados: La edad promedio de los sujetos fue de 54 años; la mayoría fueron mujeres (64\%) y la patología más frecuente fue la trombocitopenia inmunológica (60\%). Previo al procedimiento, el $23 \%$ requirió algún tipo de soporte transfusional y en el $60 \%$ la esplenectomía se utilizó como primera o segunda línea terapéutica. En cuatro casos, el procedimiento se hizo como método diagnóstico y en otros tres, no hubo información de seguimiento. El análisis de desenlaces se realizó sobre 23 pacientes. Durante los primeros 30 días posoperatorios, se presentaron complicaciones infecciosas (17.4\%) y hemorrágicas (13\%) sin ninguna muerte atribuible al procedimiento. El 78\% tuvo respuesta hematológica global y la mediana de supervivencia global fue de 133 meses (r, 108 a 157), período que fue menor entre quienes tuvieron dependencia transfusional preoperatoria $(p=0.04)$ y para quienes lograron la respuesta hematológica $(p=0.026)$. La tasa de complicaciones a largo plazo fue inferior al $10 \%$.

Conclusiones: La esplenectomía obtiene frecuencias importantes de respuesta para patologías benignas y malignas de la sangre y se corresponde con una elevada supervivencia global relacionada a una baja tasa de complicaciones a largo plazo. La supervivencia se modifica principalmente por la dependencia transfusional preoperatoria, por lo que se sugiere explorar esta variable como determinante en otros estudios.
\end{abstract}

Palabras clave: Esplenectomía, trastornos linfoproliferativos, púrpura trombocitopénica inmunológica, anemia hemolítica, supervivencia.

\begin{abstract}
Introduction: Splenectomy represents a diagnostic and therapeutic strategy for many benign and malignant hematological conditions. The following article presents the results an analyzed series of splenectomized patients due to hematological diseases in Fundación Santa Fé de Bogotá.

Materials and methods: Between 2004 and 2011, thirty patients were splenectomized based on hematological benign $(n=23)$ and malignant $(n=7)$ disease as indication. Clinical records were evaluated to obtain information on various parameters and long term outcomes.
\end{abstract}


Results: The average age of patients was 54 years old and the sample was mainly composed by women (64\%). Before surgery, the principal diagnosis was immune thrombocytopenic purpura (ITP) (60\%); $23 \%$ required any degree of transfusional support and splenectomy was used as first or second line of treatment in $60 \%$ of the cases. Seven cases were excluded: four due to diagnostic splenectomy and three due to loss to follow-up. 23 patients were included for long-term analysis. During the 30-day post-operative period, there were $17.4 \%$ infectious and $13 \%$ hemorrhagic complications, none of which led to death. A complete hematological response was obtained following splenectomy in $78 \%$ of the cases and the overall survival was 133 months ( $r, 108-157)$, which was lower in whom had transfusional dependence $(p=0.04)$, and whom reached any hematologic response $(p=0.026)$. Long-term adverse events presented in less than $10 \%$.

Conclusions: Splenectomy reaches important hematologic responses in benign and malignant blood diseases and results in a high survival rate, with a low frequency of complications. Overall survival is modified mainly by preoperative transfusional dependence; we suggest exploring this variable as a survival predictor in future studies.

Key words: Splenectomy, lymphoproliferative disorders, immunologic thrombocytopenic purpura, hemolytic anemia, survival.

\section{Introducción}

La resección quirúrgica total o parcial del bazo es un procedimiento realizado frecuentemente, lo que se refleja en las cerca de 22.000 esplenectomías practicadas en los Estados Unidos durante el 2005'. En el 44\% de los casos, esta intervención se practica de forma terapéutica, equitativamente entre las enfermedades hematológicas benignas y el trauma esplénico²; en menor grado, se utiliza en el proceso de estadificación y diagnóstico de algunas patologías malignas (20.2\% y $9.5 \%$, respectivamente) $)^{3}$.

En 1911, Sutherland reportó por primera vez una esplenectomía como tratamiento de la esferocitosis hereditaria $(E H)^{4}, y$, posteriormente, en 1916, se utilizó para controlar las manifestaciones de la púrpura trombocitopénica inmunológica (PTI) ${ }^{5}$. Desde entonces, la intervención ha seguido un patrón dinámico, consolidándose como un eje terapéutico bien caracterizado en múltiples patologías hematológicas. Dentro de las condiciones más reconocidas, se encuentran la PTI, la anemia hemolítica $(\mathrm{AH})$ de diversa índole y algunos tipos de linfomas localizados en el bazo ${ }^{6-10}$. Por ejemplo, para el caso de la PTI, después de la introducción de los corticosteroides por Dameshek en 195111, tradicionalmente se ha reportado su uso en aquellos pacientes que han sido refractarios al tratamiento. Se ha estimado que, en aquellos que no responden al manejo conservador, la resección del bazo logra la remisión completa en dos terceras partes de la población afecta ${ }^{12}$. Para los casos de las AH autoinmunes, la esplenectomía tiene una efectividad similar a la inmunoterapia cuando se ha fracasado al tratamiento con esteroides, prefiriéndose, en general, sobre esta última opción ${ }^{8}$. Para aquellos que presentan una $\mathrm{AH}$ de carácter hereditario, la esplenectomía reduce el requerimiento transfusional medio, alcanzando diversas respuestas globales de la enfermedad en presencia de una membranopatía (esferocitosis y eliptocitosis hereditaria), enzimopatía (deficiencia de piruvato kinasa) o hemoglobinopatía (talasemias) ${ }^{13,14}$.

Estudios recientes, desarrollados en pacientes con neoplasias hematológicas esplénicas (específicamente en el linfoma marginal primario del bazo), han demostrado que el procedimiento es seguro y eficaz cuando se realiza por laparoscopia, logrando una tasa de remisión completa a mediano y largo plazo que oscila alrededor del $65 \%$ al $100 \%$, incluyendo con frecuencia la combinación con quimioterapia adyuvante $\mathrm{e}^{15,16}$. Lo anterior contrasta con una tasa de complicaciones mayores y menores relacionadas con la técnica quirúrgica inferior al $23 \%$, siendo las más frecuentes las atelectasias, el neumotórax, el íleo prolongado, las complicaciones de la herida quirúrgica y los derrames pleurales ${ }^{17}$. Actualmente, el papel de la cirugía es debatible para estos casos debido a que en algunos registros se ha observado una tasa de respuesta similar con el uso de quimioinmunoterapia sin la intervención quirúrgica ${ }^{16}$.

Con el tiempo, el perfeccionamiento de las habilidades y técnicas quirúrgicas ha permitido la introducción de procedimientos mínimamente invasivos, como la laparoscopia, que ofrecen menos complicaciones, tiempo de hospitalización y morbimortalidad respecto del procedimiento efectuado a cielo abierto ${ }^{18-20}$. Esta vía fue descrita por primera vez en 1991 por Delaitre y colaboradores ${ }^{21}$, y su diseminación en la práctica clínica regular es una realidad en nuestro entorno ${ }^{22}$. A continuación, se describe una serie de pacientes esplenectomizados por alguna indicación hematológica en el Hospital Universitario de la Fundación Santa Fe de Bogotá (Colombia). 


\section{Materiales y métodos}

El objetivo principal del estudio fue determinar la supervivencia global (SG) de los pacientes esplenectomizados por alguna enfermedad hematológica en la Fundación Santa Fe de Bogotá (FSFB), y plantear posibles factores que pudieran modificarla. Los objetivos secundarios contemplaron estimar la supervivencia libre de recaída (SLR), la frecuencia de la respuesta hematológica y de las complicaciones a corto plazo y a largo plazo.

Se diseñó un estudio descriptivo retrospectivo que incluyó los pacientes esplenectomizados por cualquier técnica entre los años 2004 y 2011. El tamaño de la muestra a priori no fue calculado debido a la heterogeneidad y pequeño tamaño esperado de la población, por lo que fue determinado a conveniencia. Los registros fueron identificados a través del sistema virtual de historias clínicas, utilizando los códigos únicos CIE-10 correspondientes al procedimiento. Se incluyeron en el estudio aquellos pacientes que registraron como diagnóstico principal cualquier enfermedad hematológica, sin considerar restricciones por la edad o indicación quirúrgica, y fueron excluidos todos los casos de esplenectomía por trauma.

La información fue extraída de los registros por dos investigadores ( $A A$ y $D C$ ), quienes ingresaron los datos en una base diseñada para el estudio. Como estrategia de verificación, se seleccionaron tres variables definitorias para valorar el acuerdo entre investigadores al momento de la captura de la información: indicación del procedimiento, diagnóstico definitivo y respuesta hematológica. Sobre estas, cada investigador evaluó la concordancia de los datos extraídos por duplicado (intraobservador) y luego se comparó la información extraída por los dos investigadores (interobservador). Adicionalmente, se estimó la frecuencia de datos faltantes en las historias clínicas. Con el objetivo de mejorar el rendimiento de la información obtenida, se estableció contacto telefónico con los pacientes para corroborar fechas definitorias para el cálculo de la supervivencia y se llevó a cabo una revisión manual de los registros quirúrgicos. La digitación y evaluación se realizó en forma nominal, sin ajustar los datos de la historia clínica, usando el paquete estadístico SPSS versión 18.0 (IBM ${ }^{\circledR}$ software, Chicago, IL, EE. UU.).
Se recolectaron diversas variables clínicas (edad, sexo, líneas de tratamiento previas, dependencia transfusional -definida como el requerimiento de transfundir cualquier componente debidamente indicado en el último año antes del procedimiento quirúrgico-, número de líneas hematológicas comprometidas), los desenlaces derivados de la cirugía (técnica quirúrgica, complicaciones a 30 días y mortalidad), algunas características histopatológicas de los bazos resecados (peso, presencia y tipo de neoplasia), la evolución de la enfermedad hematológica y su respuesta (determinada a los 30 y 120 días para las patologías benignas y malignas, respectivamente), las infecciones por gérmenes encapsulados ( $N$. meningitidis y $H$. influenzae) conocidas como OPSI (overwhelming post-splenectomy infection), los eventos trombóticos al término del seguimiento, la SG y SLR.

En todos los casos, la respuesta hematológica se determinó según la enfermedad. Para la PTI, se definió respuesta completa (RC), parcial (RP) y ausente de acuerdo con el recuento plaquetario medido al menos en dos ocasiones 30 días después de la cirugía en $>100.000 \mathrm{cel} / \mathrm{ml}, 100.000-30.000 \mathrm{cel} / \mathrm{ml}$ o $<30.000 \mathrm{cel} / \mathrm{ml}$, respectivamente. Para la $\mathrm{AH}$, la RC se consideró cuando se encontró un valor de hemoglobina en rango normal para la edad sin requerimiento transfusional por al menos seis meses después de la esplenectomía; la RP, cuando hubo una mejoría de al menos el 50\% sobre el nivel basal de la hemoglobina registrado antes de la cirugía; y ausencia de respuesta, en presencia de una mejoría menor al 50\% o por la necesidad de otras intervenciones médicas (inmunoterapia y danazol, entre otros). En los pacientes con linfomas, se usaron los criterios de Cheson ${ }^{23}$ cuando se encontró enfermedad medible; en quienes se documentó compromiso de médula ósea, la respuesta se valoró con una nueva muestra que debió ser negativa tras el tratamiento.

Los resultados se caracterizaron mediante la determinación de frecuencias absolutas, relativas, medidas de tendencia central y de dispersión. Para la determinación de asociaciones entre frecuencias de variables categóricas evaluadas, se hizo un análisis univariado usando el estadístico Ji al cuadrado $\left(\mathrm{X}^{2}\right)$ o el test exacto de Fisher cuando fue necesario. Para todos los casos, el nivel de significancia fue de $p<0.05$. Por ser ajeno a la conducta terapéutica, para la determinación de las 
tasas de respuesta y supervivencia en los pacientes con neoplasias, se excluyeron del análisis los casos en los que la esplenectomía se llevó a cabo como procedimiento diagnóstico. Las estimaciones de supervivencia se hicieron tomando como fecha inicial la de la esplenectomía y como fecha final la ocurrencia de muerte (SG) o de recaída por los criterios anteriormente definidos (SLR). Se utilizó el modelo no paramétrico del límite del producto (método de Kaplan-Meier) y sus funciones se compararon mediante las pruebas del Log-rank y Tarone-Ware. Con el fin de valorar los factores que influyeron sobre la supervivencia libre de recaída y global, se realizó un análisis multivariado empleando el modelo proporcional de Cox (introducir). Las variables incluidas en dicho modelo fueron seleccionadas a partir de un consenso de especialistas en hematología, las cuales fisiopatológicamente se consideraron como modificadoras de la supervivencia y que tuvieron positividad en el análisis univariado.

\section{Resultados}

Sobre la información recolectada, se estimó un nivel de concordancia intraobservador del 100\% e interobservador del $94 \%$. Sobre el total de datos proyectados en el aplicativo, se pudo completar la información correspondiente en más del 90\%; lo anterior fue posible gracias a la disponibilidad de una historia clínica digitalizada y sistematizada, quedando datos faltantes en quienes no fue posible el seguimiento clínico posterior a la esplenectomía por motivos administrativos o de procedencia.

\section{Características de la población en estudio}

La tabla 1 muestra las características generales de los pacientes incluidos en el estudio. Un total de 30 pacientes con edades entre los 2 y 81 años fueron incluidos en el estudio (mediana de 54 años); de estos, el 63\% eran mujeres $(n=19)$. El diagnóstico dominante fue la PTI en 3 de cada 5 pacientes; de ellos, el $67 \%$ eran del sexo femenino, relación que no mostró dominancia para el resto de los diagnósticos $(p=0.94)$. Las neoplasias hematológicas (23\%) y las $\mathrm{AH}(17 \%)$ resultaron menos frecuentes. El $40 \%$ de la población había recibido dos o más líneas de tratamiento médico antes de la esplenectomía, hecho positivo para los pacientes con PTI, pero no para aquellos con $\mathrm{AH}$, donde el $80 \%$ solo se expuso a una intervención médica antes de la cirugía 16.7. La mayoría de los sujetos tenía al menos una citopenia manifiesta en el hemograma (77\%); el 13\%, dos o más; y el 10\%, ninguna. Casi una cuarta parte de la población presentó algún grado de dependencia transfusional, característica dominante entre quienes tenían $\mathrm{AH}(p=0.03)$ y sexo masculino $(p=0.02)$. Este hallazgo no fue positivo en los 16 pacientes que habían recibido dos o más tratamientos antes de la cirugía $(p=0.52)$.

En general, los últimos tratamientos recibidos antes de la esplenectomía fueron los esteroides (67\%), seguidos en frecuencia por la inmunoterapia (17\%). A lo largo de la enfermedad, 25 pacientes recibieron esteroides; 10, algún tipo de inmunoterapia (gammaglobulina humana o anti-D); y 4, rituximab. La minoría de pacientes recibió inmunosupresión (ciclosporina, micofenolato mofetil, ciclofosfamida) o danazol.

Tabla 1. Características demográficas y clínicas de los pacientes sometidos a esplenectomía por enfermedad hematológica en la FSFB entre 2004 y 2011 ( $\mathrm{n}=30$ )

\begin{tabular}{|c|c|c|c|c|}
\hline & \multicolumn{3}{|c|}{ Diagnóstico principal } & \multirow{2}{*}{ Total } \\
\hline & PTI & $\mathrm{AH}^{*}$ & Neoplasia hematológica" & \\
\hline Número de pacientes $n(\%)^{\dagger}$ & $18(60.0)$ & $5(16.7)$ & $7(23.3)$ & $30(100)$ \\
\hline Género masculino $n(\%)$ & $6(33.3)$ & $3(60)$ & $2(28.6)$ & $11(36.7)$ \\
\hline Edad mediana al diagnóstico años (rango) & $50.5(17-75)$ & $54(2-66)$ & $78(64-81)$ & $54.5(2-81)$ \\
\hline Dependencia transfusional $n(\%)$ & $1(5.6)$ & $4(80)$ & $2(28.6)$ & $7(23.3)$ \\
\hline \multicolumn{5}{|l|}{ Líneas de tratamiento previos a la esplenectomía } \\
\hline $0 \circ 1 n(\%)$ & $8(44.4)$ & $4(80)$ & $6(85.7)$ & $18(60)$ \\
\hline$\geq 2 n(\%)$ & $10(55.6)$ & $1(20)$ & $1(14.3)$ & $12(40)$ \\
\hline \multicolumn{5}{|l|}{ Citopenias en sangre periférica } \\
\hline $0 \circ 1 n(\%)$ & $17(94.4)$ & $4(80)$ & $4(57.1)$ & $25(83.3)$ \\
\hline$\geq 2 n(\%)$ & $1(5.6)$ & $1(20)$ & $3(42.9)$ & $5(16.7)$ \\
\hline Técnica laparoscópica n (\%) & $17(94.4)$ & $3(60)$ & $4(57.1)$ & $24(80)$ \\
\hline Indicación terapéutica de la esplenectomía n (\%) & $18(100)$ & $5(100)$ & $3(42.9)$ & $26(86.6)$ \\
\hline Peso mediano del bazo en gramos (rango) & $89.5(35-150)$ & $490(297-1002)$ & $505(154-2304)$ & $132.5(35-2304)$ \\
\hline \multicolumn{5}{|c|}{$\begin{array}{l}\text { "Incluye } 2 \text { pacientes con anemia hemolítica autoinmune, } 2 \text { pacientes con anemia hemolítica sin etiología determinada, } 1 \text { paciente con enzimopatía. } \\
\text { "Incluye } 3 \text { pacientes con linfoma marginal esplénico, } 1 \text { paciente con linfoma B difuso de célula grande con infiltración esplénica, } 1 \text { paciente con linfoma no primario del bazo, } 1 \text { paciente con } \\
\text { linfoma de Hodgkin de localización esplénica, } 1 \text { paciente con leucemia linfocítica crónica. } \\
{ }^{\dagger} \text { Dentro del total de todos los casos. }\end{array}$} \\
\hline
\end{tabular}


La indicación para la realización de la esplenectomía fue terapéutica predominantemente y en cerca del $15 \%$ su motivo fue diagnóstico. En la gran mayoría de los casos, el procedimiento se llevó a cabo por vía laparoscópica (80\%), requiriendo conversión en 2 de ellos (ambos casos registraron un peso medio del bazo superior a $130 \mathrm{~g})$.

La cuantificación del peso (mediana) de los bazos resecados fue de $330 \mathrm{~g}$ (r, 35-2.304). En un 70\%, se apreció congestión de la pulpa roja, siendo este hallazgo más frecuente en quienes tuvieron un peso menor a $130 \mathrm{~g}(\mathrm{p}=0.02)$ y en los que se confirmó el diagnóstico de PTI ( $p=0.02)$. Por otra parte, la hiperplasia de la pulpa blanca y la hematopoyesis extramedular se encontraron en menor grado (28\% y $7 \%$ de los casos, respectivamente), sin alguna preferencia especial según el diagnóstico. En 7 casos fue evidente la infiltración tumoral esplénica, con el hallazgo incidental de un angiosarcoma de localización focal en un paciente con PTI. En ningún espécimen se documentó fibrosis extensa ni trombosis.

\section{Desenlaces y complicaciones a corto y a largo plazo}

De los 30 pacientes esplenectomizados, 4 fueron llevados al procedimiento por indicación diagnóstica asociada a esplenomegalia: 2 en el estudio de adenomegalias sistémicas, 1 por una AH autoinmune corticorresistente y 1 por leucopenia. En los cuatro casos, se evidenció un síndrome linfoproliferativo esplénico no primario y se administró el tratamiento correspondiente. De los 26 sujetos restantes, en $3(11.5 \%)$ no fue posible realizar el seguimiento (1 paciente con $\mathrm{AH}$ autoinmune y 2 con PTI). Por lo anterior, la respuesta al tratamiento y la presentación de complicaciones fue estimada sobre 23 pacientes.

La tabla 2 exhibe la frecuencia de respuestas hematológicas y complicaciones en los primeros 30 días tras la esplenectomía, así como los desenlaces a largo plazo. En el $70 \%(n=19)$ de los casos, se alcanzó la respuesta global $(R G=R C+R P)$ de las alteraciones hematológicas tras la realización de la esplenectomía, sin una tendencia estadísticamente significativa al considerar el diagnóstico (PTI frente a los otros). Sin embargo, se obtuvo una RG en más del $80 \%$ de los casos de PTI y en las neoplasias hematológicas, no así en los casos de $\mathrm{AH}(\approx 25 \%)$. Para las neoplasias, el manejo posesplenectomía incluyó la consolidación del tratamiento con quimioterapia y/o inmunoterapia.

Respecto al período posoperatorio, el 8.3\% $(n=2)$ tuvo alguna complicación infecciosa o hemorrágica, que en ningún caso resultó en mortalidad temprana. No hubo diferencias en la presentación de complicaciones posoperatorias según el tipo de enfermedad hematológica ( $p=0.55)$, pero sí cuando se discriminó la relación según la dependencia transfusional $(p=0.0001)$ y el no alcanzar una respuesta hematológica ( $p=0.009$ ). Al término del seguimiento, todos $(n=3)$ los pacientes que tuvieron alguna complicación perioperatoria habían muerto, frente al $13.6 \%$ de los que no las presentaron $(p=0.013)$.

Tabla 2. Frecuencia de respuesta hematológica y eventos adversos a corto y largo plazo después de la esplenectomía $(n=23)$

\begin{tabular}{|c|c|c|c|c|}
\hline & \multicolumn{3}{|c|}{ Diagnóstico principal } & \multirow{2}{*}{ Total } \\
\hline & PTI & $\mathrm{AH}^{*}$ & Neoplasia hematológica" & \\
\hline \multicolumn{5}{|l|}{ Respuesta a corto plazo } \\
\hline Respuesta hematológica global (RC + RP) $n(\%)$ & $14(87.5)$ & $1(25)$ & $3(100)^{+}$ & $18(78.3)$ \\
\hline No respuesta $n(\%)$ & $2(12.5)$ & $3(100)$ & $0(0)$ & $5(21.7)$ \\
\hline \multicolumn{5}{|l|}{ Complicaciones a corto plazo } \\
\hline Infecciosas n (\%) & $1(6.3)$ & $3(75)$ & $0(0)$ & $4(17.4)$ \\
\hline Hemorrágicas $n(\%)$ & $1(6.3)$ & $1(25)$ & $1(33)$ & $3(13)$ \\
\hline Recaída a largo plazo $n(\%)^{* * *}$ & $0(0)$ & $0(0)$ & $0(0)$ & $0(0)$ \\
\hline \multicolumn{5}{|l|}{ Complicaciones a corto plazo $n(\%)$} \\
\hline Infecciosas n (\%) & $0(0)$ & $2(50)$ & $0(0)$ & $2(8.3)$ \\
\hline Trombóticas $n$ (\%) & $1(5.9)$ & $0(0)$ & $1(33)$ & $2(8.3)$ \\
\hline Mortalidad a largo plazo $n(\%)$ & $1(6.3)$ & $2(50)$ & $0(0)$ & $3(13)$ \\
\hline Pérdida del seguimiento $n(\%)$ & $2(11)$ & $1(20)$ & $0(0)$ & $3(11.5)$ \\
\hline Número de pacientes $n(\%)^{\ddagger}$ & $16(69.6)$ & $4(17.4)$ & $3(13.4)$ & $23(100)$ \\
\hline \multicolumn{5}{|c|}{$\begin{array}{l}\text { "Incluye } 1 \text { paciente con anemia hemolítica autoinmune, } 2 \text { pacientes con anemia hemolítica sin etiología determinada, } 1 \text { paciente con enzimopatía. } \\
\text { ”" Incluye } 2 \text { pacientes con linfoma marginal esplénico y } 1 \text { paciente con leucemia linfocítica crónica. } \\
\text { †" Sobre el total de pacientes que alcanzaron algún grado de respuesta hematológica. } \\
\text { †El manejo de estos pacientes incluyó quimioterapia y/o inmunoterapia en todos los casos. } \\
\text { ₹ Análisis sobre el total de pacientes con seguimiento posquirúrgico. }\end{array}$} \\
\hline
\end{tabular}




\section{Impacto clínico de la esplenectomía y supervivencia}

De los pacientes que lograron la RG con el procedimiento, este fenómeno fue mantenido durante el seguimiento y no se presentaron recaídas. Por el anterior motivo, la SLR se homologó a la SG y no fue calculada para nuestra serie. Los factores que se asociaron a una mayor SG se ilustran en la tabla 3 y fueron principalmente la dependencia transfusional $(p=0.04)$ y el logro de la respuesta hematológica $(p=0.026)$.

Al termino del estudio (mediana de seguimiento 44.7 meses), 4 pacientes (15\%) habían muerto, ninguno de ellos por la condición hematológica de base. La supervivencia global (SG) fue de 133 meses (IC95\% 108.5 a 157 meses) (figura 1), mayor para el subgrupo de las PTI (149 meses; IC95\% 128 a 170) en comparación con aquellos que tenían otras entidades nosológicas (79 meses; IC95\% 49.5 a 109.6, $\mathrm{p}=0.084$; figura $2 \mathrm{~A}$ ). La evaluación del mismo desenlace discriminado por el peso del bazo encontró

Tabla 3. Asociaciones entre la mortalidad a largo plazo en un modelo univariado para los pacientes esplenectomizados de acuerdo con diversos factores clínicos, histopatológicos e intervenciones posteriores $(n=23)$

\begin{tabular}{|c|c|c|c|}
\hline & $\begin{array}{l}\text { Número de } \\
\text { pacientes } \\
(n=23)\end{array}$ & $\begin{array}{c}\text { Mortalidad } \\
(\%)\end{array}$ & Valor $p$ \\
\hline \multicolumn{4}{|l|}{ Edad } \\
\hline Menor de 50 años & 11 & $1(9.1)$ & \multirow{2}{*}{ NS } \\
\hline Mayor de 50 años & 12 & $1(8.3)$ & \\
\hline \multicolumn{4}{|l|}{ Sexo } \\
\hline Masculino & 8 & $1(12.5)$ & \multirow{2}{*}{ NS } \\
\hline Femenino & 15 & $1(6.7)$ & \\
\hline \multicolumn{4}{|l|}{ Tipo de enfermedad hematológica } \\
\hline $\begin{array}{l}\text { Púrpura trombocitopénica } \\
\text { inmunológica }\end{array}$ & 17 & $1(5.9)$ & \multirow{3}{*}{ NS } \\
\hline Anemias hemolíticas & 3 & $1(33.0)$ & \\
\hline Enfermedades malignas & 3 & $0(0)$ & \\
\hline \multicolumn{4}{|c|}{ Número de líneas de tratamiento previo a la esplenectomía } \\
\hline $0 \circ 1$ & 12 & $0(0)$ & \multirow{2}{*}{ NS } \\
\hline$\geq 2$ & 11 & $2(18.2)$ & \\
\hline \multicolumn{4}{|c|}{ Dependencia transfusional prequirúrgica } \\
\hline Sí & 5 & $2(40.0)$ & \multirow{2}{*}{0.04} \\
\hline No & 18 & $0(0)$ & \\
\hline \multicolumn{4}{|c|}{ Peso del bazo al estudio histopatológico } \\
\hline$<130 \mathrm{~g}$ & 14 & $0(0)$ & \multirow{2}{*}{ NS } \\
\hline$\geq 130 \mathrm{~g}$ & 9 & $2(22.2)$ & \\
\hline \multicolumn{4}{|c|}{ Requerimiento de tratamiento a largo plazo } \\
\hline Sí & 5 & $1(20.0)$ & \multirow{2}{*}{ NS } \\
\hline No & 18 & $1(5.6)$ & \\
\hline \multicolumn{4}{|l|}{ Respuesta hematológica a largo plazo } \\
\hline Sí & 18 & $0(0)$ & \multirow{2}{*}{0.026} \\
\hline No & 4 & $2(50.0)$ & \\
\hline \multicolumn{4}{|l|}{ Complicaciones a largo plazo } \\
\hline Sí & 4 & $1(25)$ & NS \\
\hline No & 19 & $1(5.3)$ & \\
\hline
\end{tabular}

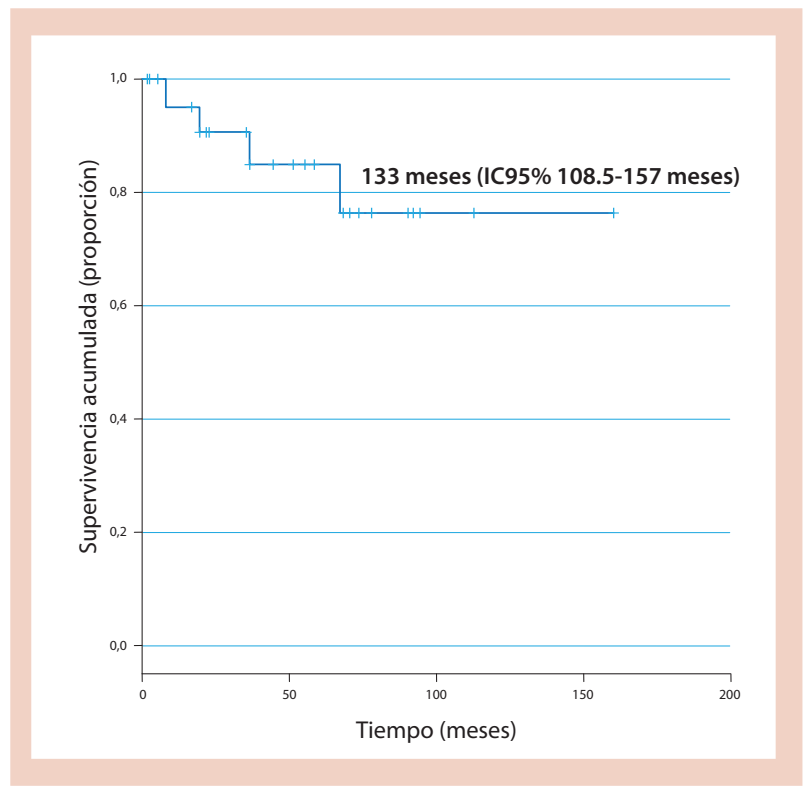

Figura 1. Supervivencia global en los pacientes $(n=23)$ con enfermedad hematológica tratados con esplenectomía en la FSFB.

una diferencia estadísticamente significativa a favor de quienes obtuvieron un valor inferior a $130 \mathrm{~g}$-no alcanzado (NA) versus 36.6 meses; $p=0.024$; figura 2B-. De forma similar, la ausencia de dependencia transfusional modificó positivamente la supervivencia (NA versus 36 meses; $p=0.001$ ), hallazgo concordante con el estimativo de las medianas conforme al grado de respuesta (en presencia de $\mathrm{RC}$, la SG no se ha alcanzado, mientras que este valor para quienes no respondieron fue 36.6 meses; $p=0.007$ ) (dato no mostrado). Los pacientes que no requirieron tratamiento posterior tuvieron una mayor SG (con y sin tratamiento posterior a la esplenectomía 51 y 152 meses, respectivamente; $p=0.007)$, relación dominante para el grupo con PTI (2/10 sujetos). Además, la presentación

Tabla 4. Modelo proporcional de Cox para las variables determinantes de la supervivencia en los pacientes esplenectomizados por enfermedades hematológicas en la FSFB $(n=23)$

\begin{tabular}{|c|c|c|c|c|}
\hline \multirow{2}{*}{ Variable } & \multirow{2}{*}{$\begin{array}{c}\text { Razón de } \\
\text { riesgo }\end{array}$} & \multicolumn{2}{|c|}{ IC95\% } & \multirow{2}{*}{ Valor $p$} \\
\hline & & Inferior & Superior & \\
\hline $\begin{array}{l}\text { Peso del bazo mayor o } \\
\text { menor de } 130 \mathrm{~g}\end{array}$ & NA & NA & NA & 0.109 \\
\hline Dependencia transfusional & 187003.63 & 0 & $8.34 E+140$ & 0.029 \\
\hline Respuesta hematológica & NA & NA & NA & 0.108 \\
\hline Sexo & 1.479 & 0.152 & 14.393 & 0.135 \\
\hline $\begin{array}{l}\text { Requerimiento de } \\
\text { tratamiento posterior }\end{array}$ & NA & NA & NA & 0.140 \\
\hline $\begin{array}{l}\text { Recepción de más de una } \\
\text { línea de tratamiento previo }\end{array}$ & NA & NA & NA & 0.892 \\
\hline $\begin{array}{l}\text { PTI frente a otros } \\
\text { diagnósticos }\end{array}$ & NA & NA & NA & 0.177 \\
\hline
\end{tabular}


Figura 2A. Supervivencia global según el tipo de diagnóstico

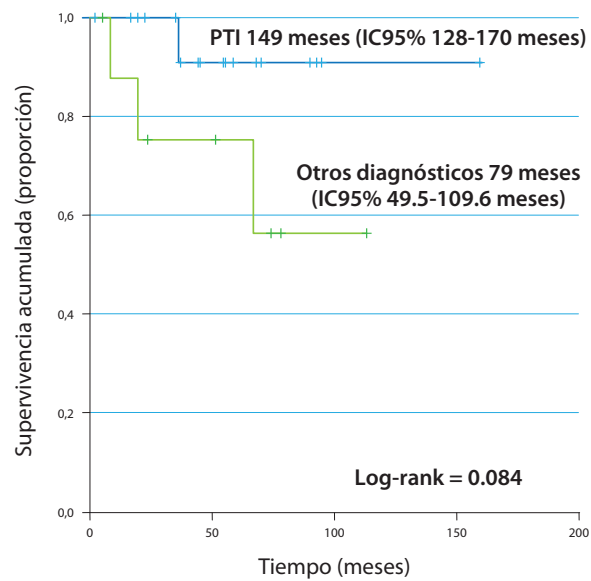

Figura 2C. Supervivencia global según la dependencia transfusional prequirúrgica

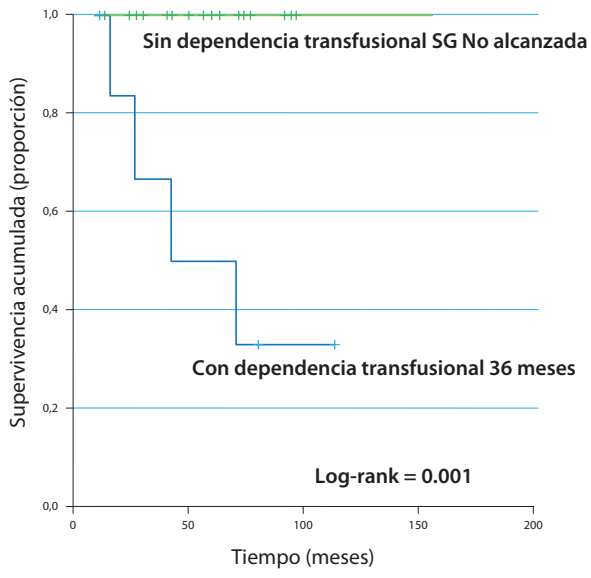

Figura 2B. Supervivencia global según el peso del bazo

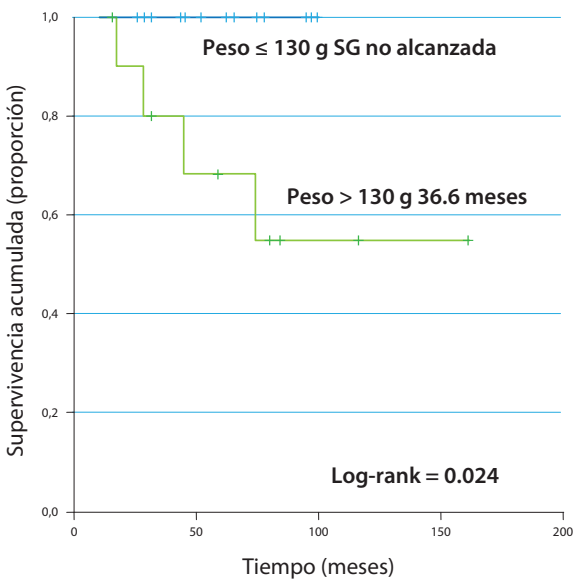

Figura 2D. Supervivencia global según el requerimiento del tratamiento posterior a la esplenectomía

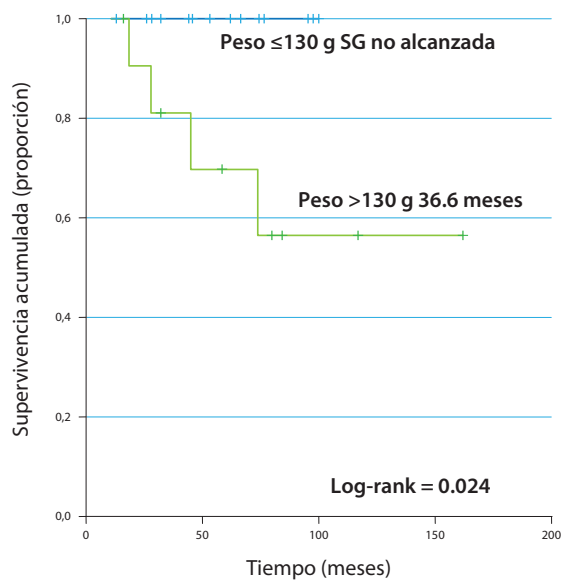

Figura 2. Supervivencia global en los pacientes esplenectomizados de acuerdo con las cuatro variables determinantes obtenidas en el modelo proporcional de Cox.
Figura 2E. Supervivencia global según la presentación de complicaciones a largo plazo

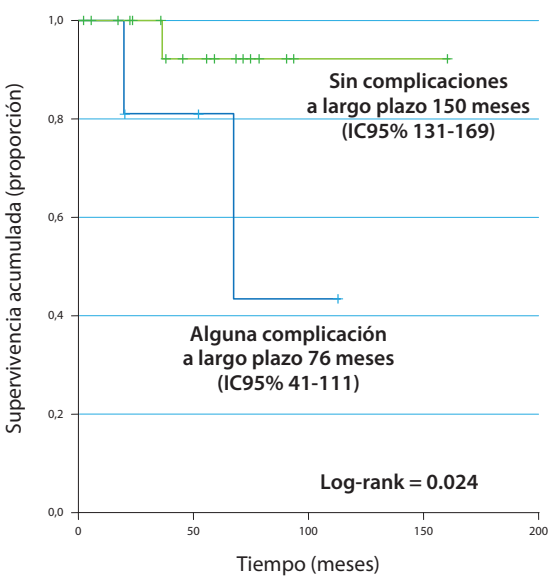


de complicaciones a largo plazo mostró cierta tendencia a disminuir la supervivencia $(p=0.062$ ). No se encontraron diferencias estadísticamente significativas para la SG respecto del número de líneas de tratamiento antes de la intervención (al menos una línea, 98 meses versus 2 líneas o más 131 meses; $\mathrm{p}=0.788$ ), según el tipo de abordaje quirúrgico (laparotomía 87 meses versus laparoscopia 135 meses; $p=0.733$ ) o la edad estratificada a partir de los 50 años (< 50 años 145 meses versus $>50$ años 78 meses; $p=0.381)$. Finalmente, el análisis multivariado solo identificó a la dependencia transfusional como variable que determinó la supervivencia (tabla 4).

\section{Discusión}

Aun después de 100 años desde su descripción, la esplenectomía continúa representando una alternativa terapéutica ampliamente considerada para los pacientes con diversas patologías hematológicas: la PTI, la púrpura trombocitopénica trombótica (PTT), las AH de diversa índole, junto con ciertas neoplasias de la sangre tanto de localización esplénica primaria como secundaria. Sin embargo, los estudios de mejor evidencia (experimentos clínicos aleatorizados y metaanálisis) son escasos o nulos respecto a esta intervención. Por otro lado, son numerosas las series de casos y cohortes (con tamaño y población variables) que han descrito la efectividad de la esplenectomía para estas condiciones, especialmente a corto plazo ${ }^{24}$ y con pocos datos del contexto latinoamericano ${ }^{22}$. En estas, es claramente predominante el papel de la PTI como la indicación más frecuente de la esplenectomía; lo anterior podría estar relacionado con su incidencia (en especial en la población femenina) cuantificada en 3.9 casos por 100.000 habitantes/año $0^{25}$. Las entidades hematológicas malignas y las $\mathrm{AH}$ le siguen en frecuencia como indicaciones del procedimiento, siendo su orden variable según el estudio 17,22,26,27. En el presente trabajo, la distribución de las indicaciones se mantuvo alrededor de lo descrito en la literatura.

Conforme con la condición hematológica, la edad al momento de la esplenectomía también es relativa; tanto en los casos de PTI y algunos de AH es notoria la afectación entre los más jóvenes ${ }^{25,28}$. Esta distribución se opone al promedio de edad de los pacientes con neoplasias hematológicas, entidades en las que se aprecia un rango superior ${ }^{18}$. Si bien la presente serie no contó con un número representativo de pacientes con AH y neoplasias, en el caso de la PTI sí fue evidente la afectación de la población femenina, acorde con lo reportado en otras series. La estratificación de los grupos según la edad y la patología en nuestra cohorte es coherente con el curso biológico de estas enfermedades, y sigue lo informado previamente ${ }^{17}$. Respecto a la esplenectomía en pacientes pediátricos, existe un acuerdo general para posponer la operación hasta que se haya alcanzado un mínimo de seis años de edad, otorgando un limitado nivel de evidencia ${ }^{2,29}$. En nuestra serie, se llevó un paciente al procedimiento a los dos años de edad por una AH documentada al nacimiento, sin causa evidente, pero con un requerimiento transfusional intensivo (cada dos semanas). Aunque no se logró obtener la respuesta hematológica, el paciente disminuyó la frecuencia e intensidad del soporte que venía recibiendo y hasta la fecha del seguimiento continuaba vivo y con menor morbilidad.

La piedra angular en el manejo inicial de múltiples condiciones hematológicas (en especial las benignas) que afectan el bazo son los corticoesteroides; su uso es explicable como consecuencia del efecto pleiotrópico sobre el sistema reticuloendotelia|30,31. De allí que la mayoría de líneas de tratamiento previas a la esplenectomía hayan sido estos agentes. Particularmente en la PTI, tras esta intervención, se recurre al procedimiento quirúrgico; en contraste y en nuestra cohorte, la esplenectomía fue diferida como tratamiento más tardío en cerca de la mitad de los pacientes con PTI o AH.

Se han hecho esfuerzos por determinar la eficacia de la esplenectomía en diversos contextos. Una revisión sistemática no convencional que incluyó 135 series de casos de más de 15 pacientes con PTI encontró una frecuencia de RC a largo plazo del 66\%; en especial, la RC después de cinco años se obtuvo en el $71 \%$ de los casos de PTI sin importar la edad ${ }^{24}$. Lo anterior contrastó con una frecuencia consolidada de complicaciones globales del $9.6 \%$ y $12.9 \%$, y de mortalidad del $1.0 \%$ y $0.2 \%$, cuando se usó la técnica laparoscópica y abierta, respectivamente. Para los casos de $\mathrm{AH}$ autoinmunes, la frecuencia de obtener una respuesta global se estimó entre el 56\% y 100\%, según su naturaleza secundaria o primaria ${ }^{8,13,32}$. En el caso de los linfomas de la zona marginal de localización esplénica, la resección del bazo ha derivado 
en tasas de respuesta superiores al $95 \%{ }^{33,34}$. No obstante, la falta de homogeneidad para indicar la esplenectomía como segunda línea de elección podría estar supeditada por la mínima cantidad de estudios clínicos de alto nivel de evidencia, que se hayan realizado a gran escala, que promulguen la efectividad del procedimiento, y por la aparición y comercialización de los nuevos agentes farmacológicos que abogan por la preservación del órgano con el fin de evitar los riesgos a los que predispone la asplenia ${ }^{7,8}$. Para ilustrar este hecho, actualmente existen estudios en curso registrados en ClinicalTrials.gov que evalúan varios agentes con el objetivo de postergar la esplenectomía en múltiples patologías hematológicas como segunda línea de manejo, tales como el anticuerpo monoclonal anti- $\mathrm{CD}_{20}$ rituximab en $\mathrm{AH}$ autoinmune (NCT01181154) y en PTI (NCT00344149), y el agonista del receptor de trombopoyetina AMG531 en PTI (NCT00415532). En el caso de las neoplasias hematológicas esplénicas, el uso de la quimioterapia y/o inmunoterapia concomitante ha resultado en tasas de respuesta similares a las obtenidas mediante la cirugía ${ }^{35,36}$. En el presente trabajo, la RG fue acorde con lo reportado en la literatura para los casos de PTI, sin embargo, para los otros, no fue posible deducir su frecuencia por el bajo número de pacientes por grupo.

La tasa de complicaciones perioperatorias fue aceptable, debido principalmente a las infecciones (del sitio operatorio, locales de otros sitios o sistémicas) que en general fueron manejadas exitosamente (no hubo ninguna muerte por sepsis) en los 30 días posoperatorios, resultando en una mortalidad temprana nula. Si bien su frecuencia fue levemente menor en nuestra serie, es fundamental resaltar que los pacientes cuya cirugía es de mayor complejidad (pacientes con esplenomegalia importante) fueron pocos. Por otro lado, la morbilidad posoperatoria se ha reportado hasta en el $23 \%$, siendo principalmente de tipo no infeccioso 20,37 y congruente con nuestros hallazgos. A largo plazo, las complicaciones de índole infecciosa y trombótica solo se presentaron cada una en un paciente. Esta frecuencia es similar a lo reportado ${ }^{38}$, y es justificable por el uso sistemático de la vacunación contra gérmenes encapsulados.

Por ser patologías crónicas, los estudios disponibles han evaluado predominantemente la SLR. Para la PTI, se ha estimado una SLR a cinco años entre el
$70 \%$ y $90 \%$, y a 10 años del $55 \%$ al $65 \%{ }^{38-42}$. Sin embargo, estos valores suelen estar comprometidos por la pérdida de pacientes durante el seguimiento, evento que oscila alrededor del 30\%, al igual que por la heterogeneidad de la población incluida en dichos estudios $^{38-40,42}$. Lo anterior explica porque los factores determinantes de la SLR han sido controvertidos en este grupo de pacientes, sugiriendo algún impacto sobre el pronóstico de la edad, el sexo, y la respuesta a los esteroides antes de la cirugía, entre otros ${ }^{24,39}$. La SG en PTI, por el contrario, ha sido pobremente caracterizada $^{38}$. En el presente estudio, la SG no pudo ser comparada con la SLR, puesto que los pacientes fueron expuestos con frecuencia a otras opciones terapéuticas posesplenectomía.

Para las AH no autoinmunes (p. ej., enzimopatías), la experiencia resulta más escasa y limitada, reportando estabilidad de los requerimientos transfusionales, en las cifras de hemoglobina y en el período libre de tratamiento, después de 1 y 8 años de seguimiento posterior a la esplenectomía13,14,43. Por tal motivo, en los pacientes con $\mathrm{AH}$ por deficiencia de piruvato-quinasa, se recomienda desde hace varias décadas practicar la esplenectomía en los jóvenes severamente afectados que requieren un soporte transfusional regular y toleran pobremente la anemia ${ }^{44}$. En nuestra serie, en una paciente de seis años con esta entidad, se logró la RC después de la cirugía, resultado que se mantuvo hasta el término del seguimiento. En los casos de AH autoinmunes, la información de supervivencia es escasa y se concentra en pequeños registros ${ }^{13} \mathrm{o}$ en estudios sobre nuevas estrategias terapéuticas ${ }^{45}$. Para los pacientes con neoplasias hematológicas de localización esplénica, la SLP ha sido estimada en pocas series después de 3 a 5 años, y oscila entre el $50 \%$ y $80 \%{ }^{33,46,47}$. Respecto de la SG, se ha encontrado entre el $70 \%$ y $90 \%$, luego de 3 y 5 años de evaluación, respectivamente. Estas cifras son inferiores a las obtenidas con el uso de quimioterapia y/o inmunoterapia ${ }^{16,36,47}$

En nuestra cohorte, los factores que resultaron determinantes de la SG presentan explicaciones plausibles. En primer lugar, la respuesta hematológica es sinónima al control de la enfermedad, por lo que es esperable que su obtención se traduzca en mejores expectativas de vida. La mejoría de la supervivencia a partir de la respuesta terapéutica también ha sido bien caracterizada en otras patologías neoplásicas 
hematológicas, tales como en el mieloma múltiple ${ }^{48}$. En segundo lugar, la relevancia de la dependencia transfusional podría explicarse desde la perspectiva de la fisiopatología de las condiciones hematológicas; el valor pronóstico de la dependencia transfusional ha sido ampliamente caracterizado en el síndrome mielodisplásico (SMD) y en la mielofibrosis, donde hace parte de las escalas pronósticas validadas internacionalmente, y es directamente proporcional a un comportamiento biológico más agresivo y a una mayor carga de la enfermedad clonal ${ }^{49-51}$. Adicionalmente, la dependencia transfusional en ciertos casos puede resultar en complicaciones crónicas que podrían impactar la SG (p. ej., la sobrecarga de hierro $)^{49}$. Algunos grupos han utilizado el análisis de la supervivencia discriminada por la intensidad y carga de los requerimientos transfusionales sobre el pronóstico en los pacientes con $\mathrm{SMD}^{52}$, no obstante, esta variable puede afectarse por la edad, el sexo, las comorbilidades extrahematológicas, el estado funcional y el rendimiento de cada unidad transfundida ${ }^{53}$. La dependencia transfusional preoperatoria no se ha considerado en general en los estudios de eficacia de la esplenectomía, por lo que nuestros resultados tendrán que confirmarse en el futuro.

Las limitaciones dominantes de este trabajo fueron la naturaleza retrospectiva del análisis que pudo promover un sesgo de información. Además, su carácter unicéntrico limitó el tamaño de la muestra, lo que puede impactar la validez externa de los resultados.
Por otra parte, el bajo número de pacientes con AH y neoplasias hematológicas podría resultar en asociaciones erráticas. La proporción de pérdida del seguimiento a largo plazo se estimó en un $10 \%$, lo que resulta aceptable. Por último, es necesario determinar una forma estandarizada para estimar el impacto de la dependencia transfusional (posiblemente según su intensidad). Lo anterior es una prioridad de trabajo de varios grupos que estudian las enfermedades hematológicas, en las que el bazo puede desempeñar un papel fisiopatológico fundamental.

\section{Conclusiones}

La esplenectomía obtiene frecuencias importantes de respuesta hematológica en patologías hematológicas benignas y malignas, lo que se corresponde con una elevada SG y una baja tasa de complicaciones a largo plazo. La supervivencia se modifica principalmente por la dependencia transfusional preoperatoria, variable que debe explorarse como determinante en otros trabajos.

\section{Agradecimientos}

Los autores desean agradecer de forma especial al doctor Milton Lombana del Departamento de Hematología y Oncología del Hospital Militar Central de Bogotá por su colaboración y aportes tras la revisión del manuscrito.

\section{Referencias}

1. DeFrances CJ, Cullen KA, Kozak LJ. National Hospital Discharge Survey: 2005 annual summary with detailed diagnosis and procedure data. Vital Health Stat. 2007:(165):1-209.

2. Di Sabatino A, Carsetti R, Corazza GR. Post-splenectomy and hyposplenic states. Lancet. 2011;378(9785):86-97.

3. Kraus MD, Fleming MD, Vonderheide RH. The spleen as a diagnostic specimen: a review of 10 years' experience at two tertiary care institutions. Cancer. 2001;91(11):2001-9.

4. Sutherland GA, Burghard FF. The Treatment of Splenic Anaemia by Splenectomy. Proc R Soc Med. 1911;4(Clin Sect):58-70.

5. Kaznelson P. Splenogene thrombolytische purpura. Wien Klin Wochn Schr. 1916:29:1451-4.

6. Miwa S. Pyruvate kinase deficiency. Prog Clin Biol Res. 1990;344:843-52

7. Neunert C, Lim W, Crowther M, Cohen A, Solberg L Jr, Crowther MA, et al. The American Society of Hematology 2011 evidence- based practice guideline for immune thrombocytopenia. Blood. 2011;117(16):4190-207.

8. Lechner K, Jäger U. How I treat autoimmune hemolytic anemias in adults. Blood. 2010;116(11):1831-8.

9. Schilling RF. Risks and benefits of splenectomy versus no splenectomy for hereditary spherocytosis--a personal view. Br J Haematol. 2009;145(6):728-32.

10. Taher A, Isma'eel H, Cappellini MD. Thalassemia intermedia: revisited. Blood Cells Mol Dis. 2006;37(1):12-20.

11. Dameshek W, Rubio F Jr, Mahoney JP, Reeves WH, Burgin LA. Treatment of idiopathic thrombocytopenic purpura (ITP) with prednisone. J Am Med Assoc. 1958;166(15):1805-15.

12. Stasi R, Provan D. Management of immune thrombocytopenic purpura in adults. Mayo Clin Proc. 2004;79(4):504-22.

13. Coon WW. Splenectomy in the treatment of hemolytic anemia. Arch Surg. 1985;120(5):625-8 
14. Necheles TF, Finkel HE, Sheehan RG, Allen DM. Red cell pyruvate kinase deficiency. The effect of splenectomy. Arch Intern Med. 1966;118(1):75-8.

15. Iannitto E, Ambrosetti A, Ammatuna E, Colosio M, Florena AM, Tripodo C, et al. Splenic marginal zone lymphoma with or without villous lymphocytes. Hematologic findings and outcomes in a series of 57 patients. Cancer. 2004;101(9):2050-7.

16. Bennett M, Schechter GP. Treatment of splenic marginal zone lymphoma: splenectomy versus rituximab. Semin Hematol. 2010;47(2):143-7.

17. Berman RS, Yahanda AM, Mansfield PF, Hemmila MR, Sweeney JF, Porter GA, et al. Laparoscopic splenectomy in patients with hematologic malignancies. Am J Surg. 1999;178(6):530-6.

18. Cavaliere D, Torelli P, Panaro F, Casaccia M, Ghinolfi D, Santori $\mathrm{G}$, et al. Outcome of laparoscopic splenectomy for malignant hematologic diseases. Tumori. 2004;90(2):229-32.

19. Rosen M, Brody F, Walsh RM, Tarnoff M, Malm J, Ponsky J. Outcome of laparoscopic splenectomy based on hematologic indication. Surg Endosc. 2002;16(2):272-9.

20. Franklin M, Gálvez-Hernández CA, Treviño J, Villegas-Cabello O, González-Ruvalcaba R, Díaz-Elizondo J. [Laparoscopic splenectomy: Twelve-year experience in two private institutions]. Cir Cir. 2006;74(6):443-7.

21. Delaitre B, Maignien B. [Splenectomy by the laparascopic approach. Report of a case]. Presse Med. 1991;20(44):2263.

22. Maluenda GF, P PB, Braghetto MI, Csendes JA. Esplenectomía laparoscópica en enfermedades hematológicas. Rev Med Chile. 2004;132:189-94.

23. Cheson BD, Pfistner B, Juweid ME, Gascoyne RD, Specht L, Horning SJ, et al. Revised response criteria for malignant lymphoma. J Clin Oncol. 2007;25(5):579-86

24. Kojouri K, Vesely SK, Terrell DR, George JN. Splenectomy for adult patients with idiopathic thrombocytopenic purpura: a systematic review to assess long-term platelet count responses, prediction of response, and surgical complications. Blood. 2004;104(9):2623-34.

25. Schoonen WM, Kucera G, Coalson J, Li L, Rutstein M, Mowat $F$, et al. Epidemiology of immune thrombocytopenic purpura in the General Practice Research Database. Br J Haematol. 2009;145(2):235-44.

26. Balagué $C$, Targarona EM, Cerdán G, Novell J, Montero O Bendahan $G$, et al. Long-term outcome after laparoscopic splenectomy related to hematologic diagnosis. Surg Endosc. 2004;18(8):1283-7.

27. Musser G, Lazar G, Hocking W, Busuttil RW. Splenectomy for hematologic disease. The UCLA experience with 306 patients. Ann Surg. 1984;200(1):40-5.

28. Ucar K. Clinical presentation and management of hemolytic anemias. Oncology. (Williston Park) 2002;16(9 Suppl 10):163-70.

29. Habermalz B, Sauerland S, Decker G, Delaitre B, Gigot JF, Leandros $E$, et al. Laparoscopic splenectomy: the clinical practice guidelines of the European Association for Endoscopic Surgery (EAES). Surg Endosc. 2008;22(4):821-48.

30. Garratty G. The James Blundell Award Lecture 2007: do we really understand immune red cell destruction? Transfus Med. 2008;18(6):321-34

31. Atkinson JP, Schreiber AD, Frank MM. Effects of corticosteroids and splenectomy on the immune clearance and destruction of erythrocytes. J Clin Invest. 1973;52(6):1509-17.
32. Akpek G, McAneny D, Weintraub L. Comparative response to splenectomy in Coombs-positive autoimmune hemolytic anemia with or without associated disease. Am J Hematol. 1999;61(2):98-102

33. Thieblemont $C$, Felman $P$, Berger $F$, Dumontet $C$, Arnaud $P$, Hequet $\mathrm{O}$, et al. Treatment of splenic marginal zone B-cell lymphoma: an analysis of 81 patients. Clin Lymphoma. 2002;3(1):41-7.

34. Troussard X, Valensi F, Duchayne E, Garand R, Felman P, Tulliez $M$, et al. Splenic lymphoma with villous lymphocytes: clinical presentation, biology and prognostic factors in a series of 100 patients. Groupe Francais d'Hematologie Cellulaire (GFHC). Br J Haematol. 1996;93(3):731-6.

35. Kalpadakis C, Pangalis GA, Dimopoulou MN, Vassilakopoulos TP, Kyrtsonis MC, Korkolopoulou P, et al. Rituximab monotherapy is highly effective in splenic marginal zone lymphoma. Hematol Oncol. 2007;25(3):127-31.

36. Arcaini L, Orlandi E, Scotti M, Brusamolino E, Passamonti F, Burcheri $\mathrm{S}$, et al. Combination of rituximab, cyclophosphamide, and vincristine induces complete hematologic remission of splenic marginal zone lymphoma. Clin Lymphoma. 2004;4(4):250-2.

37. Zimmer J, Andrés E, Noel E, Koumarianou A, Blicklé JF, Maloisel F. Current management of adult idiopathic thrombocytopenic purpura in practice: a cohort study of 201 patients from a single center. Clin Lab Haematol. 2004;26(2):137-42.

38. Kumar S, Diehn FE, Gertz MA, Tefferi A. Splenectomy for immune thrombocytopenic purpura: long-term results and treatment of postsplenectomy relapses. Ann Hematol. 2002;81(6):312-9.

39. Kaya E, Erkurt MA, Aydogdu I, Kuku I, Ozhan O, Oner RI, et al. Retrospective analysis of patients with idiopathic thrombocytopenic purpura from Eastern Anatolia. Med Princ Pract. 2007;16(2):100-6.

40. Schwartz J, Leber MD, Gillis S, Giunta A, Eldor A, Bussel JB. Long term follow-up after splenectomy performed for immune thrombocytopenic purpura (ITP). Am J Hematol. 2003;72(2):94-8.

41. Wang $T, X u$ M, Ji L, Han ZC, Yang R. Splenectomy for adult chronic idiopathic thrombocytopenic purpura: experience from a single center in China. Eur J Haematol. 2005;75(5):424-9.

42. Pamuk GE, Pamuk ON, Baslar Z, Ongören S, Soysal T, Ferhanoglu B, et al. Overview of 321 patients with idiopathic thrombocytopenic purpura. Retrospective analysis of the clinical features and response to therapy. Ann Hematol. 2002;81(8):436-40.

43. Gordon-Smith EC. Erythrocyte enzyme deficiencies. Pyruvate kinase deficiency. J Clin Pathol Suppl. (R Coll Pathol) 1974;8:128-33.

44. Tanaka KR, Paglia DE. Pyruvate kinase deficiency. Semin Hematol. 1971;8(4):367-96.

45. Dierickx D, Verhoef $G$, Van Hoof A, Mineur P, Roest A, Triffet $A$, et al. Rituximab in auto-immune haemolytic anaemia and immune thrombocytopenic purpura: a Belgian retrospective multicentric study. J Intern Med. 2009;266(5):484-91.

46. Mulligan SP, Matutes E, Dearden C, Catovsky D. Splenic lymphoma with villous lymphocytes: natural history and response to therapy in 50 cases. Br J Haematol. 1991;78(2):206-9.

47. Tsimberidou AM, Catovsky D, Schlette E, O'Brien S, Wierda WG, Kantarjian $\mathrm{H}$, et al. Outcomes in patients with splenic marginal zone lymphoma and marginal zone lymphoma treated with rituximab with or without chemotherapy or chemotherapy alone. Cancer. 2006;107(1):125-35

48. Chanan-Khan AA, Giralt S. Importance of achieving a complete response in multiple myeloma, and the impact of novel agents. J Clin Oncol. 2010:28(15):2612-24. 
49. Malcovati L, Porta MG, Pascutto C, Invernizzi R, Boni M, Travaglino $\mathrm{E}$, et al. Prognostic factors and life expectancy in myelodysplastic syndromes classified according to WHO criteria: a basis for clinical decision making. J Clin Oncol. 2005;23(30):7594603.

50. Pardanani A, Tefferi A. Prognostic relevance of anemia and transfusion dependency in myelodysplastic syndromes and primary myelofibrosis. Haematologica. 2011;96(1):8-10.

51. Elena C, Passamonti F, Rumi E, Malcovati L, Arcaini L, Boveri E, et al. Red blood cell transfusion-dependency implies a poor survival in primary myelofibrosis irrespective of IPSS and DIPSS. Haematologica. 2011;96(1):167-70.

52. Pereira A, Nomdedeu M, Aguilar JL, Belkaid M, Carrió A, Cobo $F$, et al. Transfusion intensity, not the cumulative red blood cell transfusion burden, determines the prognosis of patients with myelodysplastic syndrome on chronic transfusion support. Am J Hematol. 2011;86(3):245-50.

53. Malcovati L, Cazzola M. The relevance of transfusion-dependency in the prognostic assessment of patients with myeloid neoplasms. Am J Hematol. 2011;86(3):241-3. 